Laser Chem., 1999, Vol. 19, pp. 191-194 Reprints available directly from the publisher Photocopying permitted by license only
(C) 1999 OPA (Overseas Publishers Association) N.V. Published by license under the Harwood Academic Publishers imprint, part of The Gordon and Breach Publishing Group.

\title{
THE RESONANCE RAMAN SPECTRA OF BIOLOGICALLY RELEVANT POLYENE DERIVATIVES IN $T_{1}$ : A THEORETICAL INVESTIGATION
}

\author{
FABRIZIA NEGRI* and GIORGIO ORLANDI \\ Dipartimento di Chimica “G. Ciamician”, Università di Bologna, \\ Via F. Selmi, 2, 40126 Bologna, Italy
}

(Received 21 April 1997)

\begin{abstract}
The vibrational structure of the $T_{1}$ resonance Raman spectra of $\beta$-ionylidene acetaldehyde ( $\mathrm{C} 15$ aldehyde) and retinal are analyzed with the help of semiempirical quantum chemical calculations. The $T_{1}$ equilibrium structures and vibrational force fields, along with the structure in the $T_{n}$ state which is in resonance with the exciting wavelength, are obtained and employed to model the spectra under the assumption of a dominant Franck-Condon mechanism.
\end{abstract}

Keywords: Resonance Raman spectra; simulations; semi-empirical calculations; triplet states; retinal

The polyenic structure of biochemically important chromophores, for instance carotenoids, has the dual function of light-harvesting and photo-protection of the photosynthetic systems to which the chromophores belong. In the latter function, the lowest triplet state of the polyene is involved. Thus, the investigation of the properties of this state are of particular interest and importance.

In recent years we have studied the structure and spectroscopy of the lowest triplet state of short polyenes and polyene-derivatives by performing quantum-chemical calculations followed by simulations of the spectra [1]. Here we report the results of the simulations of the

*Corresponding author. 
Resonance Raman (RR) spectra of trans C15-aldehyde and retinal in the lowest triplet state.

Since the Franck-Condon (FC) mechanism is the main source of intensity, the vibrational structure of the $T_{1}$ RR spectra discussed in the following is dominated by the activity of totally symmetric (TS) modes. To simulate the spectra we adopted the harmonic approximation and obtained, for each $i$-th totally symmetric mode, the displacement parameters $B_{i}$ relative to the $T_{1}-T_{n}$ transition.

$$
B_{i}=\left(\frac{\omega_{i}}{\hbar}\right)^{1 / 2} Q_{i\left(T_{1}\right)}^{T_{n} T_{1}}
$$

Here $Q_{i\left(T_{1}\right)}^{T_{n} T_{1}}$ is the projection of the geometry change between the two states, expressed in Cartesian coordinates, onto the $T_{1}$-state normal coordinate $Q_{i}^{T_{1}}$.

Under the assumption of resonance with the $0-0$ band of the $T_{1} \rightarrow T_{n}$ transition, the activity of each totally symmetric mode is related to the displacement parameter through the $\gamma_{i}$ parameter, that is $I_{i} \propto \gamma_{i}=(1 / 2) B_{i}^{2}$.

Thus, the molecular parameters required to simulate the RR spectra are the equilibrium structures of the two states involved in the transition, $T_{1}$ and $T_{n}$, and the vibrational force field in $T_{1}$. These were computed with an updated version [2, 3] of the semiempirical QCFF/ PI hamiltonian [4].

The results of the simulations of the triplet RR spectrum of trans C15-aldehyde and retinal are shown in Figures 1 and 2, respectively. The RR spectrum of Figure 1 can be compared with the observed spectrum of C15-aldehyde reported in Ref. [5]. The agreement is satisfactory and especially the triplet of bands observed in the $1100-$ $1300 \mathrm{~cm}^{-1}$ region is nicely reproduced by the calculations. A cursory comparison of the spectrum of Figure 2 with the experimental spectra available in the literature $[6,7]$ shows that the bands computed at 951 , $1015,1153,1218,1263,1277,1334,1375,1426,1493,1553$ and $1599 \mathrm{~cm}^{-1}$ match well the bands observed at 942, 1007, 1185, 1211, $1256,1298,1339,1379,1422,1515,1553$ and $1599 \mathrm{~cm}^{-1}$. We notice that the computed intensity is overestimated in the $1200-1300 \mathrm{~cm}^{-1}$ region, but this appears to be a typical feature of the QCFF/PI method. In view of the large dimension of these molecules, the 


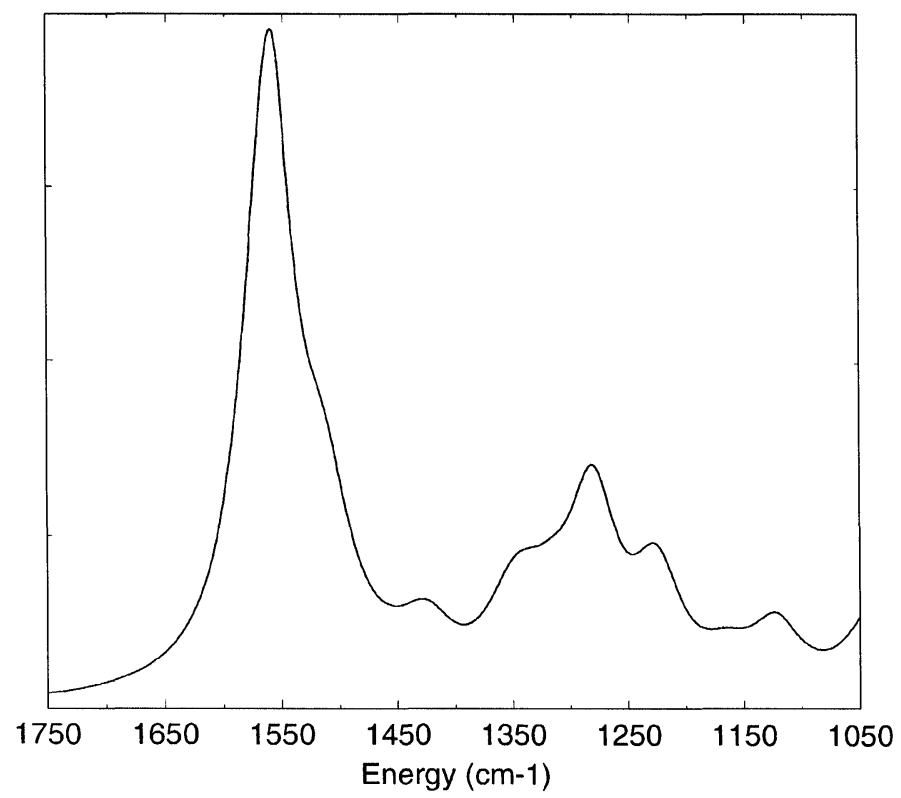

FIGURE 1 Simulation of the low resolution $T_{1}$ resonance Raman spectrum of the trans form of $\beta$-ionylidene acetaldehyde (C15 aldehyde).

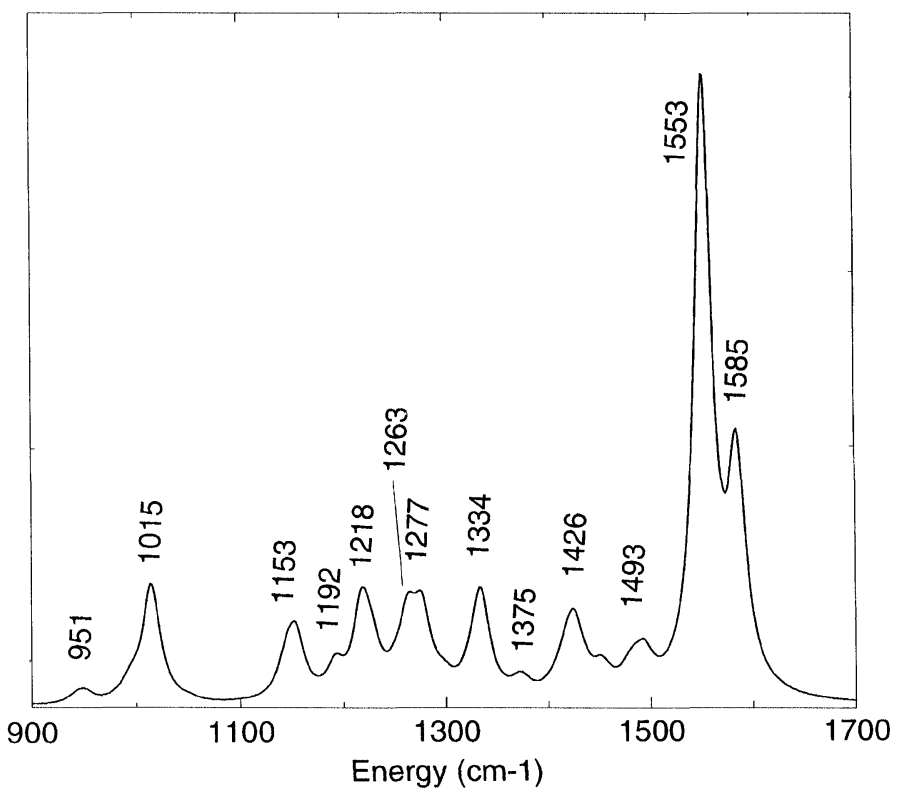

FIGURE 2 Simulation of the high resolution $T_{1}$ resonance Raman spectrum of transretinal. 
agreement between observed and simulated spectra is encouraging and suggests that the methods employed in this study can be extended to other isomeric forms of retinal and its homologues and to investigate the dynamics of the lowest triplet state.

\section{References}

[1] Negri, F. and Orlandi, G. (1995). J. Chem. Phys., 103, 2412; (1997). J. Photochem. Photobiol. A: Chem., 105, 209.

[2] Negri, F., Orlandi, G., Brouwer, A. M., Langkilde, F. W. and Wilbrandt, R. (1989). J. Chem. Phys., 90, 5944.

[3] Negri, F. and Orlandi, G. (1996). J. Phys. B: At. Mol. Opt. Phys., 95, 5049.

[4] Warshel, A. and Karplus, M. (1972). J. Am. Chem. Soc., 94, 5612.

[5] Mukai, Y., Hashimoto, H. and Koyama, Y. (1990). J. Phys. Chem., 94, 4042.

[6] Wilbrandt, R. and Jensen, N.-H. (1981). J. Am. Chem. Soc., 103, 1036; Atkinson, G. H., Pallix, J. B., Freedman, T. B., Gilmore, D. A. and Wilbrandt, R. (1981). J. Am. Chem. Soc., 103, 5069.

[7] Hamaguchi, H., Okamoto, H., Tasumi, M., Mukai, Y. and Koyama, Y. (1984). Chem. Phys. Letters., 107, 355; Mukai, Y., Abe, M., Katsuta, Y., Tomozoe, S., Ito, M. and Koyama, Y. (1995). J. Phys. Chem., 99, 7160. 